\title{
Photoacoustic Tomography in a Reflecting Cavity
}

\author{
B.T. Cox ${ }^{a}$, B. Holman ${ }^{b}$, and L. Kunyansky ${ }^{b}$ \\ ${ }^{a}$ Department of Medical Physics and Bioengineering, University College London, \\ Gower Street, London WC1E 6BT, UK \\ ${ }^{b}$ Department of Mathematics, University of Arizona, Tucson, Arizona 85721, USA.
}

\begin{abstract}
Almost all known photoacoustic image reconstruction algorithms are based on the assumption that the acoustic waves leave the object (the imaged region) after a finite time. This assumption is fulfilled if the measurements are made in free space and reflections from the detectors are negligible. However, when the object is surrounded by acoustically hard detectors arrays (and/or by additional acoustic mirrors), the acoustic waves will bounce around in such a reverberant cavity many times (in the absence of absorption, forever). This paper proposes fast reconstruction algorithms for the measurements made from the walls of a rectangular reverberant cavity. The algorithms are tested using numerical simulations.
\end{abstract}

\section{INTRODUCTION}

The interest in biomedical photoacoustic imaging has grown rapidly in the last decade or so as it provides a useful complement to existing imaging methods, from microscopic to clinical scales. Photoacoustic scanning microscopy can be used to image to very high spatial resolution (optical resolution) but only at very shallow depths. Photoacoustic (and closely related thermoacoustic) tomography, ${ }^{7,8,13,16}$ on the other hand, can achieve acoustic resolution at depths where purely optical imaging methods degrade rapidly due to optical scattering. The photoacoustic effect refers to the phenomenon whereby a pulse of sound is generated when a sufficiently short (or sufficiently rapidly modulated) pulse of light is absorbed. Because the acoustic (ultrasonic) waves that are generated are much less scattered than the light, photoacoustic tomography can provide images in optically scattering media - such as soft tissue - that are based on optical contrast but have acoustic (therefore much higher) resolution. An image with an optical rather than acoustic contrast has the potential to carry chemical information, giving photoacoustic tomography additional appeal.

\section{PHOTOACOUSTIC TOMOGRAPHY IN FREE SPACE}

In photoacoustic tomography, time domain measurements of the photoacoustically generated broadband pulses are made by an array of ultrasound detectors positioned on a measurement surface. Ideally this surface completely envelops the region of interest, as then all the available data will be collected, e.g. the detectors are placed on a spherical surface surrounding the sample to be imaged. Once the data has been collected, it must be processed before it can be displayed as an image. This step is called image reconstruction. Several idealizing assumptions are typically made to make this inverse problem solvable. First, the measurements lie on a surface completely enclosing the region of interest (as just mentioned). Second, the sound speed is the same everywhere and is known. Third, there are no acoustic reflections, including from the measurement surface. In other words, the propagation occurs as if there were nothing in the way (free space propagation) and it continues travelling out to infinity. The measurement surface is therefore not a boundary but just a set of observation points within the domain outside the support of the image region (the object).

Under these assumptions the acoustic pressure $p=p(x, t)$ of the wave propagating through free space $\left(\mathbb{R}^{n}\right.$, $n=2$ or 3 ) is a solution to the following initial value problem (IVP):

$$
\begin{aligned}
\left(\partial_{t t}-c_{0}^{2} \Delta\right) p & =0, \quad x \in \mathbb{R}^{n}, \quad t \in[0, \infty), \\
\left.p\right|_{t=0} & =p_{0},\left.\quad \partial_{t} p\right|_{t=0}=0
\end{aligned}
$$

Send correspondence to B.T. Cox, b.cox@ucl.ac.uk

Photons Plus Ultrasound: Imaging and Sensing 2013, edited by Alexander A. Oraevsky, Lihong V. Wang, Proc. of SPIE Vol. 8581, 85811D · C 2013 SPIE · CCC code: 1605-7422/13/\$18 · doi: 10.1117/12.2003571 
where $p_{0}=p_{0}(x)$ is called the initial acoustic pressure distribution. Given time series measurements of the acoustic pressure at each point of the measurement surface $S$ surrounding the region of interest $\Omega$ (within which $p_{0}$ is supported)

$$
g=p(x, t) \quad x \in S, \quad t \in[0, \mathcal{T}],
$$

it is possible to reconstruct $p_{0}$ exactly. Indeed, since $S$ is not a physical boundary but just a set of locations where pressure is measured by acoustically small detectors (not disturbing the wave), for a sufficiently large time $\mathcal{T}$, the pressure $p$ in $\Omega$ will vanish. (In $3 \mathrm{D}$, due to the Huygens principle, the pressure will vanish after $\mathcal{T}=\frac{1}{c_{0}} \operatorname{diam}(\Omega)$. In $2 \mathrm{D}$, the pressure does not completely vanish in finite time; however, it will decay fast enough that it can approximately set to 0 at sufficiently large value of $\mathcal{T} \geq \frac{1}{c_{0}} \operatorname{diam}(\Omega)$ )

Now one can solve in $\Omega$ the following initial/boundary value problem (IBVP)

$$
\begin{aligned}
\left(\partial_{t t}-c_{0}^{2} \Delta\right) p & =0, \quad t \in[0, \mathcal{T}], \quad x \in \Omega, \\
\left.p\right|_{t=\mathcal{T}} & =0,\left.\quad \partial_{t} p\right|_{t=\mathcal{T}}=0, \quad p(x, t)=g(x, t), \quad x \in S,
\end{aligned}
$$

backwards in time (from $t=\mathcal{T}$ to $t=0$ ), thus obtaining $p_{0}=p(x, 0)$. This technique is called time reversal. It is theoretically exact and works for an arbitrary closed surface $S$; many of the existing reconstruction methods are based on this idea. For instance, one can solve IBVP $(3,4)$ directly using numerical techniques. ${ }^{5,6,18}$ Eigenfunction decomposition techniques ${ }^{1,11}$ and some of the known inversion formulas ${ }^{12}$ yield reconstructions that are theoretically equivalent to the ones obtained by time reversal. In each case the crucial underlying assumption is that the values of the pressure are recorded until the acoustic wave vanishes within $\Omega$.

\section{PHOTOACOUSTIC TOMOGRAPHY IN A REFLECTIVE CAVITY}

Practically, there are a number of ways in which photoacoustic measurements can be made. One method is to scan a (small) detector from point to point, sequentially recording the acoustic pressure time series at each point. In this case the assumption of free space propagation is reasonable, as the reflection for a single detector is negligible. Frequently, in order to significantly speed up the data acquisition, instead of a single detector an array of detectors is used. Such an array will typically form a solid surface which reflects sound waves back into the object. (Both piezoelectric and optically-scanned arrays have been used.)

In the case of a single planar array the traditional reconstruction methods still apply, since the reflected waves will propagate away from the detector and will not affect the measurements. However, the images reconstructed from measurements made using a single finite-sized planar array configuration will contain 'partial view' or 'limited data' artifacts because the acoustic waves travelling close to perpendicular to the surface are not measured. In order to improve the reconstruction one could either reflect those waves back on the detector array using passive reflectors, ${ }^{3}$ or add new perpendicular detector arrays. When those new detector arrays are also reflecting, a reverberant cavity is formed. Wave propagation in a reverberant cavity is no longer represented by the IVP (1); a different mathematical model is needed.

The proper model should take into account that the domain $\Omega$ is surrounded by a reflecting boundary $\partial \Omega$, and the corresponding boundary conditions should be imposed on the wave equation (as opposed to the free space propagation discussed in the previous section). The measurement surface $S$ is now a subset of the boundary $\partial \Omega$.

When the boundary is treated as sound-hard (unity pressure reflection coefficient), the acoustic pressure $p(x, t)$ is a solution to the following initial boundary value problem:

$$
\begin{aligned}
\left(\partial_{t t}-c_{0}^{2} \Delta\right) p & =0, \quad x \in \Omega \\
p \mid(0, x) & =p_{0}, \quad \partial_{t} p(0, x)=0, \\
\partial_{n} p(x) & =0, \quad x \in \partial \Omega .
\end{aligned}
$$

Now the measurements can be written as

$$
g=p(t, x) \quad x \in S \subseteq \partial \Omega, \quad t \in[0, T] .
$$


Other boundary conditions might be appropriate in some circumstances, such as for instance if the cavity were built as a tank and one part of the boundary was a water-air interface where $p \approx 0$ (i.e. the Dirichlet conditions are imposed on this part of the boundary). Also, it is not necessary to measure the pressure on the whole boundary $\partial \Omega$, and so $S$ is only a subset of $\partial \Omega$. (Taking this to an extreme, Cox et al. ${ }^{4}$ proposed using a single measurement point when the cavity is ray-chaotic. This scheme, however, is unlikely to yield a stable reconstruction.)

A distinct property of this model is the preservation of the acoustic energy trapped within the cavity. Since the model assumes that there is no absorption of the acoustic energy by the medium, and the (Neumann or Dirichlet) boundary conditions correspond to a complete reflection of waves, the oscillations within $\Omega$ will (theoretically) continue forever. In practice, of course, this is not the case and the waves will soon decrease to the extent that further measurements will become impossible due to the low signal-to-noise ratio. While we are not explicitly modeling the absorption, we have to assume that the measurement time $T$ is bounded. It will be chosen to correspond, roughly, to several bounces of the acoustic wave between the cavity walls.

\section{IMAGE RECONSTRUCTION FROM REVERBERATION DATA}

The preservation of acoustic energy within the reverberating cavity makes it impossible to solve the inverse problem by the time reversal technique described in Section 2. Indeed, in order to obtain the accurate reconstruction of $p_{0}$ one has to accurately prescribe conditions $p(x, T)$, and $\partial_{t} p(x, T)$ to initialize the time reversal. However, there is no way to measure these data within the object. Moreover, this values are of the same order of magnitude as $p_{0}$, and if one simply replaces them by a zero (as we can safely do in the free space case) the induced error will be also of the same magnitude as $p_{0}$ we seek to reconstruct. Therefore, almost none of the known reconstruction algorithms are applicable here (with the exception of ${ }^{2-4,17}$ ). Below we propose inversion techniques suitable for photoacoustic tomography within the reverberant cavity.

\subsection{Solving the forward problem}

One can get a better insight into the problem by expanding the solution of the forward IBVP (5-7) in the series of eigenmodes of the Laplacian with Neumann boundary conditions. In other words, $p(x, t)$ can be written as

$$
p(x, t)=\sum_{m} a_{m} \varphi_{m}(x) \cos \left(\omega_{m} t\right)
$$

where $a_{m}$ and $\omega_{m}$ are the modal amplitudes and frequencies, and the mode shapes (eigenfunctions) $\varphi_{m}(x)$ satisfy the Helmholtz equation with Neumann boundary conditions:

$$
\left(\Delta+k_{m}^{2}\right) \varphi_{m}=0, \quad x \in \Omega \quad \partial_{n} \varphi_{m}=0, \quad x \in \partial \Omega .
$$

In terms of these modes, the initial pressure distribution can be written as

$$
p_{0}(x)=\sum_{m} a_{m} \varphi_{m}(x)
$$

(Here, for simplicity we have indexed the eigenmodes $\varphi_{m}(x)$ by a single index $m$. In most practical cases a more convenient indexing is by double indices in $2 \mathrm{D}$ (e.g. $\left.\varphi_{k, l}(x)\right)$ and by triple indices in 3D (e.g. $\varphi_{k, l, m}(x)$ ).) As the shape of the cavity $\Omega$ is known, the mode shapes and frequencies, $\varphi_{m}$ and $k_{m}$, can be precalculated, and the image reconstruction then reduces to estimating the modal amplitudes $a_{m}$ from the measured data $g(x, t)$ :

$$
g(x, t)=\sum_{m} a_{m} \varphi_{m}(x) \cos \left(\omega_{m} t\right) w(t), \quad x \in S, \quad t \in[0, T] .
$$

One of the simplest geometries for the cavity $\Omega$ is a square, in $2 \mathrm{D}$, or a cube, in $3 \mathrm{D}$. This geometry is also the most important from the practical point of view since it naturally arises when several square planar detector arrays are placed around the region of interest. In what follows we will consider the $2 \mathrm{D}$ case, a square 
domain, for simplicity. The $3 \mathrm{D}$ case is very similar. In the case when domain is a square $(0,1) \times(0,1)$, the Neumann eigenmodes $\varphi_{k, l}(x)$ are just products of cosines:

$$
\varphi_{k, l}(x)=\cos \left(\pi k x_{1}\right) \cos \left(\pi l x_{2}\right), \quad x=\left(x_{1}, x_{2}\right) \in \Omega=(0,1) \times(0,1) .
$$

We will assume, for simplicity and without loss of generalisation, that throughout the rest of the paper the speed of sound $c_{0}=1$. Then the eigenfrequencies $\omega_{k, l}$ can be easily found:

$$
\omega_{k, l}=\pi \sqrt{k^{2}+l^{2}}, \quad k, l=0,1,2, \ldots
$$

Now

$$
p(x, t)=\sum_{k=0}^{\infty} \sum_{l=0}^{\infty} a_{k, l} \cos \left(\pi k x_{1}\right) \cos \left(\pi l x_{2}\right) \cos \left(\omega_{k, l} t\right), \quad x \in \Omega, \quad t \in[0, T] .
$$

Below we discuss image reconstruction from the data measured either on one or on several sides of a square domain.

\subsection{Reconstruction from the data measured on one side}

Our goal is to reconstruct the coefficients $a_{k, l}$ from the values of $p(x, t)$ known on one or more sides of the square $\Omega$. Once $a_{k, l}$ 's are found, the initial pressure distribution $p(x, 0)$ is obtained by summing the Fourier series:

$$
p_{0}(x)=p(x, 0)=\sum_{k=0}^{\infty} \sum_{l=0}^{\infty} a_{k, l} \cos \left(\pi k x_{1}\right) \cos \left(\pi l x_{2}\right) .
$$

Let us first analyze the data measured on one side of the square (say, the one where $x_{1}=0$ ):

$$
\left.g_{1}\left(x_{2}, t\right) \equiv p(x, t)\right|_{x_{1}=0}=\sum_{k=0}^{\infty} \sum_{l=0}^{\infty} a_{k, l} \cos \left(\pi l x_{2}\right) \cos \left(\omega_{k, l} t\right), \quad t \in[0, T] .
$$

Due to the orthogonality of the cosine functions (in variable $x_{2}$ ) the above equation splits:

$$
\begin{aligned}
\sum_{l=0}^{\infty} a_{k, l} \cos \left(\omega_{k, l} t\right) & =g_{1, k}(t), \quad t \in[0, T], \quad k=0,1,2, \ldots \\
g_{1, k}(t) & \equiv 2 \int_{0}^{1} g_{1}\left(x_{2}, t\right) \cos \left(\pi k x_{2}\right) d x_{2} .
\end{aligned}
$$

It follows from Eq. (10) that each of the $k$ functions $g_{1, k}(t)$ is a combination of cosine functions in $t$ with known frequencies; the values $a_{k, l}$ can be viewed as generalized Fourier coefficients. More precisely, Eq. (10) can be re-written as an inverse Fourier transform $\mathcal{F}^{-1}$ of a sequence of Dirac delta-functions:

$$
g_{1, k}(t)=\sqrt{2 \pi} \mathcal{F}^{-1}\left[\sum_{l=0}^{\infty} a_{k, l} \frac{\delta\left(\xi-\omega_{k, l}\right)+\delta\left(\xi+\omega_{k, l}\right)}{2}\right],
$$

where the forward and inverse Fourier transform of an arbitrary function $h(t)$ are defined as follows

$$
\begin{aligned}
\hat{h}(\xi) & \equiv(\mathcal{F} h)(\xi) \equiv \frac{1}{\sqrt{2 \pi}} \int_{\mathbb{R}} h(t) e^{-i \xi t} d t \\
h(t) & \equiv\left(\mathcal{F}^{-1} \hat{h}\right)(t) \equiv \frac{1}{\sqrt{2 \pi}} \int_{\mathbb{R}} \hat{h}(\xi) e^{i \xi t} d \xi
\end{aligned}
$$


One can conclude that the Fourier transform $\hat{g}_{1, k}(\xi)$ of $g_{1, k}(t)$ is the following distribution

$$
\hat{g}_{1, k}(\xi)=\sqrt{2 \pi} \sum_{l=0}^{\infty} a_{k, l} \frac{\delta\left(\xi-\omega_{k, l}\right)+\delta\left(\xi+\omega_{k, l}\right)}{2}
$$

It is tempting to try to recover coefficients $a_{k, l}$ by applying the Fourier transform $\mathcal{F}$ to each $g_{1, k}(t)$ and thus obtaining $\hat{g}_{1, k}(\xi)$. However, functions $g_{1, k}(t)$ do not vanish at infinity, and they (in general) are not periodic on any finite interval $[0, T]$. Therefore, such a computation would be quite inaccurate, at best.

A technique well known in digital signal processing as a way to Fourier transform long time series, is to multiply the signal by a window function. For convenience, let us extend $g_{1, k}(t)$ as an even function to the interval $[-T, T]$ (formulas (9) and (10) will remain valid under such extension). Consider now an even, infinitely smooth function $\eta(t)$ vanishing with all its derivatives at -1 and 1 , and its scaled version $\eta_{T}(t)=\eta(t / T)$. Since the product $\eta_{T}(t) g_{1, k}(t)$ is periodic on $[-T, T]$, its Fourier transform

$$
\widehat{\eta_{T} g_{1, k}}(\xi) \equiv \frac{1}{\sqrt{2 \pi}} \int_{-T}^{T} \eta_{T}(t) g_{1, k}(t) e^{-i \xi t} d t
$$

can be easily computed (for example, by applying the composite trapezoid rule to the discretized signal). Now, by the convolution theorem

$$
\widehat{\eta_{T} g_{1, k}}(\xi)=\frac{1}{\sqrt{2 \pi}} \widehat{\eta_{T}}(\xi) * \widehat{g_{1, k}}(\xi)=\frac{1}{\sqrt{2 \pi}} \int_{\mathbb{R}} \widehat{\eta_{T}}(\zeta-\xi) \widehat{g_{1, k}}(\zeta) d \zeta
$$

and, taking into account (12),

$$
\widehat{\eta_{T} g_{1, k}}(\xi)=\sum_{l=0}^{\infty} a_{k, l} \frac{\widehat{\eta_{T}}\left(\xi-\omega_{k, l}\right)+\widehat{\eta_{T}}\left(\xi+\omega_{k, l}\right)}{2} .
$$

Function $\widehat{\eta_{T}}(\xi)$ is infinitely smooth (as a Fourier transform of a finitely supported function), and vanishes at infinity faster than any rational function in $\xi$ (since $\eta(t)$ is infinitely smooth). One may view the problem of reconstruction of $\hat{g}_{1, k}(\xi)$ from $\widehat{\eta_{T}}(\xi) * \widehat{g_{1, k}}(\xi)$ as a deconvolution problem. Due to the smoothness of the convolution kernel $\widehat{\eta_{T}}(\xi)$ such a deconvolution is an extremely ill-posed problem. Fortunately, the problem at hand can be solved without resorting to standard deconvolution techniques. Note that we know a priori that the $\hat{g}_{1, k}(\xi)$ is a combination of delta-functions whose positions in frequency space are known (see Eq. (12)); we only need to find coefficients $a_{k, l}$. Let us assume, reasonably, that the initial pressure $p_{0}$ is essentially a band-limited function, i.e. there exists a number $N$ such that all coefficients $a_{k, l}$ vanish if $k>N$ or $|l|>N$. Let us also assume that we compute the Fourier transform (13) for all frequencies $\omega_{k, m}$ with $k, m \in[0, N]$. Then, for each fixed $k$ we obtain the following system of linear equations

$$
\widehat{\eta_{T} g_{1, k}}\left(\omega_{k, m}\right)=\sum_{l=0}^{N} a_{k, l} \frac{\widehat{\eta_{T}}\left(\omega_{k, m}-\omega_{k, l}\right)+\widehat{\eta_{T}}\left(\omega_{k, m}+\omega_{k, l}\right)}{2}, \quad m=0, \ldots, N .
$$

The matrix $A^{(k)}$ of this system has size $(N+1) \times(N+1)$, with entries $\alpha_{m, l}^{(k)}=\frac{1}{2}\left[\widehat{\eta_{T}}\left(\omega_{k, m}-\omega_{k, l}\right)+\widehat{\eta_{T}}\left(\omega_{k, m}+\omega_{k, l}\right)\right]$. Theoretically, one could try to solve systems (14) for each $k=0, \ldots, N$, find $a_{k, l}$ and, thus, reconstruct $p_{0}(x)$ from measurements made on one side of the square. This is not an optimal approach for several reasons. First, while we have not proved the invertibility of the matrices $A^{(k)}$, it is clear that for large values of $k$ they are extremely ill-conditioned (see analysis below). In addition, a direct solution of these systems would be quite expensive computationally. In what follows we find an easily computable, approximate solution valid in the asymptotic limit $T \rightarrow \infty$, and investigate its behaviour for moderate values of $T$. 
Let us analyze the structure of the matrices $A^{(k)}$. Note that

$$
\widehat{\eta_{T}}(\xi) \equiv \frac{1}{\sqrt{2 \pi}} \int_{-T}^{T} \eta(t / T) e^{-i \xi t} d t=\frac{T}{\sqrt{2 \pi}} \int_{-1}^{1} \eta(s) e^{-i \xi T s} d s=T \widehat{\eta}(T \xi) .
$$

Since $\widehat{\eta}(\xi)$ decreases faster than any rational function, for any positive integer $M$ there is a constant $B_{M}$ such that

$$
|\widehat{\eta}(\xi)| \leq \frac{B_{M}}{1+|\xi|^{M}}, \quad|T \widehat{\eta}(T \xi)| \leq \frac{T B_{M}}{1+|T \xi|^{M}}
$$

and therefore, as $T$ is increased, the non-diagonal entries $\alpha_{m, l}^{(k)}$ of $A^{(k)}$ will be decreasing faster than $\mathcal{O}\left(T^{-(M-1)}\right)$ for any $M>1$ :

$$
\begin{aligned}
2\left|\alpha_{m, l}^{(k)}\right| & =\left|\widehat{\eta_{T}}\left(\omega_{k, m}-\omega_{k, l}\right)+\widehat{\eta_{T}}\left(\omega_{k, m}+\omega_{k, l}\right)\right|=T\left|\widehat{\eta}\left(T\left(\omega_{k, m}-\omega_{k, l}\right)\right)+\widehat{\eta}\left(T\left(\omega_{k, m}+\omega_{k, l}\right)\right)\right| \\
& \leq B_{M}\left(\frac{T}{1+T^{M}\left|\omega_{k, m}-\omega_{k, l}\right|^{M}}+\frac{T}{1+T^{M}\left|\omega_{k, m}+\omega_{k, l}\right|^{M}}\right) \leq 2 B_{M} \frac{T}{1+T^{M}\left|\omega_{k, m}-\omega_{k, l}\right|^{M}} .
\end{aligned}
$$

On the other hand, the diagonal entries $\alpha_{l, l}^{(k)}$ grow as $\mathcal{O}(T)$ :

$$
2\left|\alpha_{l, l}^{(k)}\right|=\left|\widehat{\eta_{T}}(0)+\widehat{\eta_{T}}\left(2 \omega_{k, l}\right)\right|=T\left|\widehat{\eta}(0)+\widehat{\eta}\left(T\left(\omega_{k, m}+\omega_{k, l}\right)\right)\right| \geq T\left|\widehat{\eta}(0)-\frac{B_{M}}{1+T^{M}\left|2 \omega_{k, l}\right|^{M}}\right| .
$$

This implies that for sufficiently large acquisition times $T$, the matrices $A^{(k)}$ become diagonally dominant and therefore stably invertible. So in order to solve the corresponding linear systems fast, we will just approximate the $A^{(k)}$ matrices by diagonal matrices with entries equal to $T \widehat{\eta}(0)$, and compute $a_{k, l}$ by the approximate formula

$$
a_{k, l} \approx 2 \frac{\widehat{\eta_{T} g_{1, k}}\left(\omega_{k, l}\right)}{\widehat{\eta_{T}}(0)}, \quad l=0, \ldots, N
$$

This gives rise to an approximate algorithm (let's call it Algorithm A) that is stable and fast, but not very accurate. The numerical simulations presented below show noticeable artifacts resulting from such a technique. In the next section we find the cause of these artifacts and show that the method can be improved upon if more measurements are done.

\subsection{Reconstruction from the data measured on two perpendicular sides}

In the previous section we have shown at the limit $T \rightarrow \infty$ the matrices $A^{(k)}$ become approximately diagonal and the solution of the systems (14) can be approximated by formula (16). However, our numerical experiments suggest that such a reconstruction technique produces noticeable artifacts for moderate values of $T$. This happens because the decrease of the off-diagonal entries of $A^{(k)}$ depends on the lower bound of the relative difference of frequencies $\omega_{k, m}$ and $\omega_{k, l}$ (see Eq. (15)). For the simplest of these systems, corresponding to $k=0$, the eigenfrequencies grow linearly

$$
\omega_{0, m}=\pi m,
$$

and the bound (15) for the off-diagonal terms of $A^{(0)}$ has a simple form

$$
\left|\alpha_{m, l}^{(0)}\right| \leq \frac{T B_{M}}{1+T^{M}\left|\omega_{0, m}-\omega_{0, l}\right|^{M}}=\frac{T B_{M}}{1+T^{M} \pi^{M}|m-l|^{M}} .
$$

We observe that for each row of the matrix the off-diagonal elements decrease proportionally to their distance from the diagonal, and the rate of decrease does not depend on $m$. In fact, one can easily obtain a uniform (in $m$ ) bound on the sum of the off-diagonal elements:

$$
\sum_{\substack{l=0 \\ j \neq m}}^{\infty}\left|\alpha_{m, l}^{(0)}\right| \leq \sum_{\substack{l=-\infty \\ j \neq m}}^{\infty}\left|\alpha_{m,|l|}^{(0)}\right| \leq \sum_{\substack{l=-\infty \\ j \neq m}}^{\infty} \frac{T B_{M}}{1+T^{M} \pi^{M}|m-l|^{M}} \leq 2 \sum_{j=1}^{\infty} \frac{T B_{M}}{1+T^{M} \pi^{M}|l|^{M}}=\mathcal{O}\left(\frac{1}{T^{M-1}}\right) .
$$


However, when we consider the modes corresponding to large values of $k$ (and corresponding matrices $A^{(k)}$ ), the eigenfrequencies are clustered toward the value $\pi k$ from above. The smallest difference between consecutive eigenfrequencies is that between $\omega_{k, 0}$ and $\omega_{k, 1}$ :

$$
\omega_{k, 1}-\omega_{k, 0}=\pi\left(\sqrt{k^{2}+1}-\sqrt{k^{2}}\right)=\pi k\left(\sqrt{1+1 / k^{2}}-1\right) \approx \frac{\pi}{2 k} .
$$

This means that for larger values of $k$ matrices $A^{(k)}$ require higher and higher values of $T$ to become approximately diagonal; for moderate values of $T$ the use of formula (16) yields significant errors. (Moreover, matrices $A^{(k)}$ become ill-conditioned). This make image reconstruction difficult if measurements are made only on one side of the square (for moderate values of $T$ ).

On the other hand, when $m$ and $l$ are of the order of $k$ or larger, the difference between $\omega_{k, l}$ and $\omega_{k, m}$ is bounded from below. For example,

$$
\omega_{k, k+1}-\omega_{k, k}=\pi\left(\sqrt{k^{2}+(k+1)^{2}}-\sqrt{2 k^{2}}\right)=\sqrt{2} \pi k\left(\sqrt{1+(2 k+1) / 2 k^{2}}-1\right) \approx \frac{\sqrt{2} \pi}{2} .
$$

For larger values of $m$ and $l$ this difference grows, and asymptotically, for $m \gg k$ and $l \gg k$ we have $\omega_{k, l}-\omega_{k, m} \approx$ $\pi(l-m)$. This implies that the lower right block of $A^{(k)}$ (obtained by removing first $k$ rows and columns) is approximately diagonally dominant, and one can use formula (16) to approximately reconstruct half of the required coefficients

$$
a_{k, l} \approx 2 \frac{\widehat{\eta_{T} g_{1, k}}\left(\omega_{k, l}\right)}{\widehat{\eta_{T}}(0)}, \quad l=0, \ldots, N, \quad l \geq k .
$$

In order to reconstruct the second half of the coefficients $a_{k, l}$ we will assume that an additional measurement is made on the side of the square corresponding to $x_{2}=0$. All of the discussion of the previous section can be repeated with minor changes in notation; the most important change is that the roles of $k$ and $l$ are interchanged. The application of this technique yields a good approximation to the second half of the coefficients $a_{k, l}$, corresponding to $k \geq l$. We will call this method Algorithm B. It yields significantly better results than those of Algorithm A. (For free space propagation, the use of perpendicular arrays was suggested by Paltauf. ${ }^{14}$ )

\subsection{Numerical realization}

Both Algorithms, A and B, can easily be generalized to the 3D case; Algorithm B for 3D would require measurements made from three adjacent sides of a cuboid. In 3D, numerical complexity becomes a major issue for reconstruction algorithms since on a fine reconstruction mesh the number of unknowns can easily reach a hundred million or more. The proposed algorithms were designed to be fast. The important steps of the Algorithm A are (i) computing $g_{1, k}$ from the measurements (Eq. (11)), (ii) computing the Fourier transform of $\eta_{T}(t) g_{1, k}(t)$ (Eq. (13)) for non-uniformly spaced values of $\xi=\omega_{k, l}$, (iii) finding $a_{k, l}$ using formula (16), and (iv) computing $p_{0}$ by summing Fourier series (8). Let us assume that the reconstruction is to be computed on an $n \times n$ grid, there are $\mathcal{O}(n)$ detectors, and each of them measures $\mathcal{O}(n)$ time steps. Step (i) can be computed using the Fast Cosine Transform (FCT), it requires $\mathcal{O}\left(n^{2} \log n\right)$ floating points operations. Step (ii) may be computed by a trapezoid rule separately for each required value of $\xi=\omega_{k, l}$. This is how both algorithms are currently implemented; this results in $\mathcal{O}\left(n^{3}\right)$ operation count. A better approach is to use the Non-Equally Spaced Fast Fourier Transform (NSFFT) ${ }^{15}$ This is a fast, stable, and accurate numerical technique; its use will allow us to complete this step in only $\mathcal{O}\left(n^{2} \log n\right)$ flops. Step (iii) is clearly fast $\left(\mathcal{O}\left(n^{2}\right)\right.$ flops), and step (iv) can be performed using 2D FCT. Total operation count for the Algorithm A is therefore $\mathcal{O}\left(n^{2} \log n\right)$ (if NSFFT is used) for $n^{2}$ unknowns. The complexity of the Algorithm B is similar. The 3D realization of these techniques would need $\mathcal{O}\left(n^{3} \log n\right)$ flops (again, if NSFFT were used) to recover $n^{3}$ unknowns representing the 3D image. 


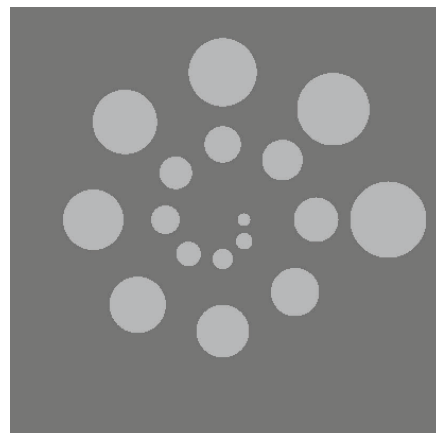

(a)

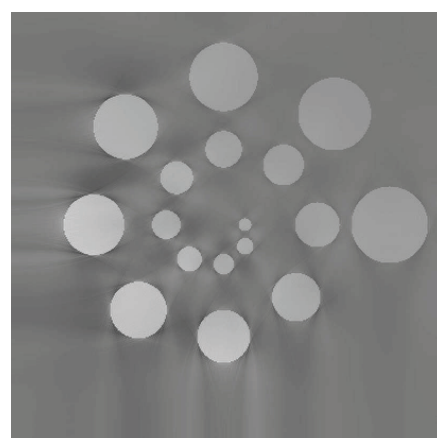

(c)

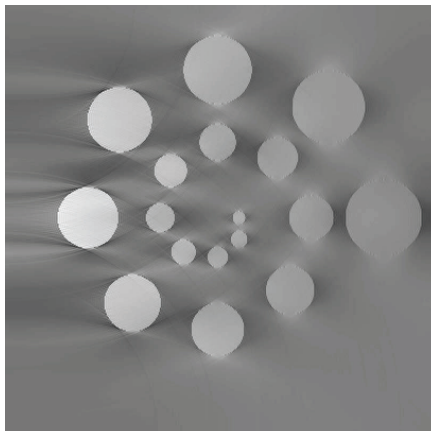

(b)

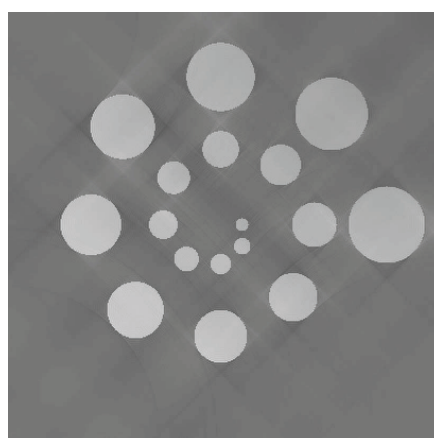

(d)

Figure 1. Example of reconstruction from simulated data $(T=2)$ : (a) phantom (b) algorithm A, data measured from the left side (c) average of two images reconstructed from the left side data and the lower side data by algorithm A (d) algorithm B, reconstruction from the data corresponding to the lower and the left sides of the square

\section{SIMULATIONS}

We illustrate Algorithms A and B by a series of numerical simulations. As a phantom representing initial pressure $p_{0}$, we use a set of characteristic functions of circles with different diameters, shown in Figure 1(a). The corresponding solution of the wave equation in a square cavity of size 1 was simulated in the interval $[0, T]$ with $T=2$. Since the simulated speed of sound is equal to $1, T=2$ corresponds to the time required for a sound wave to propagate from one wall to the opposite wall and back. This interval is quite short, since the time needed for a signal from every point to reach every detector equals $\sqrt{2}$. Figure $1(\mathrm{~b})$ shows the image reconstructed on a $401 \times 401$ grid by Algorithm A applied to the data measured on the left side of the square $\Omega$. One can see significant horizontal artifacts; besides that some of the horizontal parts of the disks' boundaries are blurred.

These distortions arise because the matrices $A^{(k)}$ are not almost-diagonal for moderate values of $T$ and large values of $k$. One can try to apply Algorithm A consecutively to the data corresponding to two adjacent sides, and then average the result. However, as Figure 1(c) demonstrates, the artifacts do not cancel. Finally, in 1(d) we present the image reconstructed by applying Algorithm B to the data corresponding to the left and lower sides of the square. This image is much closer to the phantom we seek to reconstruct, although one can notice some diagonally running artifacts. In addition, the brightness of the disks in the figure is too high in the lower left part and too low in the upper right part of the image. However, the boundaries of the circular shapes are reconstructed very well.

Figure 2 confirms our intuition and theoretical conclusion that an increase of the measurement time $T$ should significantly improve the image reconstructed by Algorithm A, and only would only marginally improve the results obtained by Algorithm B. (To reiterate, this happens since the convergence is not uniform in the 


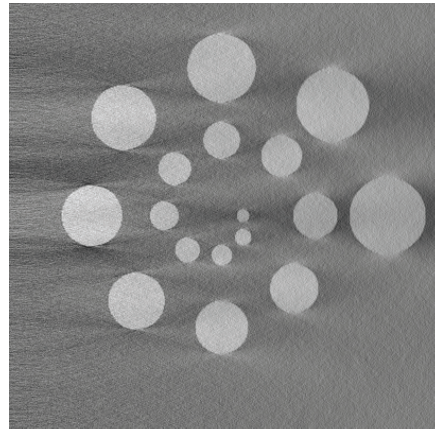

(a)

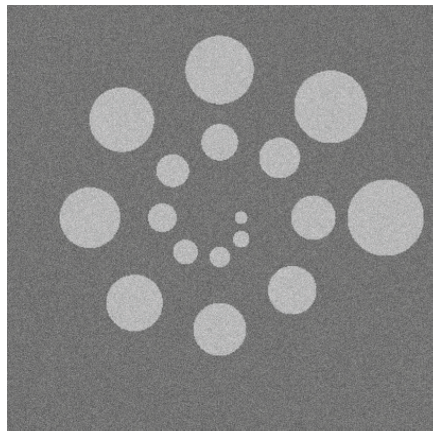

(b)

Figure 2. Reconstruction from simulated data $(T=4)$ with added 50 noise (a) Algorithm A, the left side data (b) Algorithm B, data from the left and lower sides of the square

latter case of Algorithm B: for any time $T$ there are matrices $A^{(k)}$ that are not close to being diagonal). Figure $2(\mathrm{a})$ shows the image computed by Algorithm A $(T=4)$, from the data containing $50 \%$ normally distributed noise (as measured in $L_{2}$ norm). Although there is some improvement compared to the part (b) of Figure 1, the artifacts are clearly visible, in spite of significant level of noise in the reconstructed image. In contrast, if there are any algorithm-related artifacts in the image obtained by Algorithm B (see Figure 2(b)), they are much less than the artifacts produced by noise in the data.

An interesting property of the present algorithms is a very low sensitivity to noise. Most tomography modalities tend to amplify noise, and in presence of $50 \%$ noise either the reconstructed image would be overwhelmed by noise-related artifacts, or a significant blurring would occur due to the low-frequency filtration needed to regularize the reconstruction. This does not happen in here (see, for example, Figure 2(b)) because the singularities of the solution $p(x, t)$ do not get smoothed on their way to the detectors due to the properties of the wave equation, in general, and the initial conditions of the problems (1-2) or (5-7), in particular. Such low sensitivity to noise was observed previously ${ }^{9,10}$ in other inverse problems related to PAT/TAT.

\section{DISCUSSION AND CONCLUSIONS}

Two fast and stable approximate algorithms are proposed for the problem of PAT/TAT reconstruction from the data measured on one or two walls of a square reverberant cavity. The approximation is valid in the asymptotic limit $T \rightarrow \infty$. Our numerical simulations show that both proposed methods are very stable with respect to the noise in the data. Algorithm B uses data from two adjacent walls; it yields very good approximations for quite moderate values of $T$ (e.g., $T=4$, which corresponds to four bounces of the acoustic wave between the cavity walls).

Further work is needed to find some theoretical error bounds for these methods. In order to improve the image obtained for even smaller measurement times $T$, more sophisticated techniques are needed. This is a subject of our current work. We are also currently working on generalizing the present methods for the full 3D problem, with the goal of obtaining a fast algorithm (with complexity of $O\left(n^{3} \log n\right)$ flops) capable of efficiently handling the 3D case, where the number of unknowns is expected to be greater by two or three orders of magnitude.

\section{ACKNOWLEDGMENTS}

The work of B.T. Cox was supported by the Engineering and Physical Sciences Research Council, UK, and the work of L. Kunyansky was partially supported by the NSF grants DMS-1211521 and DMS-0908243. 


\section{REFERENCES}

1. M. Agranovsky and P. Kuchment, Uniqueness of reconstruction and an inversion procedure for thermoacoustic and photoacoustic tomography with variable sound speed, Inverse Problems 23 (2007) 2089-102.

2. H. Ammari, E. Bossy, V. Jugnon, and H. Kang, Mathematical Modeling in Photoacoustic Imaging of Small Absorbers, SIAM Review 52(4) (2010) 677-95.

3. B. T. Cox, S. R. Arridge, and P. C. Beard, Photoacoustic tomography with a limited-aperture planar sensor and a reverberant cavity, Inverse Problems, 23(6) (2007) S95-S112.

4. B. T. Cox and P. C. Beard, Photoacoustic tomography with a single detector in a reverberant cavity, J. Acoust. Soc. Am., 125(3), (2009) 1426-36.

5. D. Finch, S. K. Patch, and Rakesh, Determining a Function from Its Mean Values Over a Family of Spheres, SIAM J. Math. Anal., 35(5), (2004) 1213-1240.

6. Y. Hristova, P. Kuchment, and L. Nguyen, On reconstruction and time reversal in thermoacoustic tomography in homogeneous and non-homogeneous acoustic media, Inverse Problems, 24 (2008) 055006

7. R. A. Kruger, P. Liu, Y. R. Fang, and C. R. Appledorn, Photoacoustic ultrasound (PAUS) reconstruction tomography, Med. Phys., 22 (1995) 1605-09.

8. R. A. Kruger, D. R. Reinecke, and G. A. Kruger, Thermoacoustic computed tomography - technical considerations, Med. Phys. 26 (1999) 1832-7.

9. P. Kuchment and L. Kunyansky, 2D and 3D reconstructions in acousto-electric tomography, Inverse Problems, 27, (2011) 055013.

10. L. Kunyansky, A mathematical model and inversion procedure for Magneto-Acousto-Electric Tomography (MAET), Inverse Problems, 28 (2012) 035002.

11. L. Kunyansky, A series solution and a fast algorithm for the inversion of the spherical mean Radon transform, Inverse Problems, 23 (2007) S11-S20.

12. L. Kunyansky, Reconstruction of a function from its spherical (circular) means with the centers lying on the surface of certain polygons and polyhedra, Inverse Problems, 27 (2011) 025012.

13. A. A. Oraevsky, S. L. Jacques, R. O. Esenaliev, and F. K. Tittel, Laser-based photoacoustic imaging in biological tissues, Proc. SPIE, 2134A (1994) 122-8.

14. G. Paltauf, R. Nuster, M. Haltmeier, and P. Burgholzer, Experimental evaluation of reconstruction algorithms for limited view photoacoustic tomography with line detectors, Inverse Problems, 23 (2007) S81-S94.

15. A. Dutt and V. Rokhlin, Fast Fourier Transforms For Nonequispaced Data, Siam J. Sci. Comput., 14(6) (1993) 1368-93.

16. Wang L V (Editor) 2009 "Photoacoustic imaging and spectroscopy" (CRC Press).

17. L. V. Wang, and X. Yang, Boundary conditions in photoacoustic tomography and image reconstruction, J. Biomed. Opt., 12(1) (2007) 014027

18. M. Xu and L.-H. V. Wang, Time Reversal and Its Application to Tomography with Diffracting Sources, Phys. Rev. Lett., 92(3) (2004) 3-6. 